\title{
Anabases
}

ANABASES Traditions et réceptions de l'Antiquité

18 | 2013

Varia

\section{Le centenaire du Catalogue des sculptures du Musée de Constantinople de Gustave Mendel (1912-1914)}

Annick Fenet

\section{(2) OpenEdition}

Journals

Édition électronique

URL : http://journals.openedition.org/anabases/4373

DOI : 10.4000/anabases.4373

ISSN : 2256-9421

Éditeur

E.R.A.S.M.E.

Édition imprimée

Date de publication : 1 octobre 2013

Pagination : 227-237

ISSN : 1774-4296

\section{Référence électronique}

Annick Fenet, «Le centenaire du Catalogue des sculptures du Musée de Constantinople de Gustave

Mendel (1912-1914) », Anabases [En ligne], 18 | 2013, mis en ligne le 01 novembre 2016, consulté le 21 octobre 2019. URL : http://journals.openedition.org/anabases/4373; DOI : 10.4000/anabases.4373

(c) Anabases 


\title{
Le centenaire du Catalogue des sculptures du Musée de Constantinople de Gustave Mendel (1912-1914)
}

ANNICK Fenet

\begin{abstract}
Alors que toute une série de manifestations et publications scientifiques est en préparation pour commémorer le prochain et triste centenaire du Premier conflit mondial, il n'est pas inutile de s'attarder sur les belles réalisations culturelles de l'avantguerre. Parmi celles-ci figure le Catalogue des sculptures grecques, romaines et byzantines des Musées impériaux, réalisé par Gustave Mendel et paru à Constantinople en trois volumes entre 1912 et 1914, qui reste encore aujourd'hui l'ouvrage de référence pour l'étude de la sculpture antique venue de tout l'Empire ottoman et conservée au musée archéologique d'Istanbul. Outre son intérêt intrinsèque, cette publication comporte de nombreuses particularités : une volonté manifeste de l'inscrire dans la longue durée par des notices érudites et illustrées; la collaboration franco-turque qui voit ainsi un auteur français valoriser le patrimoine de l'Asie Mineure et une institution turque ; le tout dans un contexte de transformation du monde oriental, et de rivalités occidentales en action sur le terrain asiatique à travers la pratique de l'archéologie ${ }^{1}$.
\end{abstract}

1 Sur ce thème, on lira avec profit les actes du colloque publiés par V. KRINGs et I. TASSIGNON, Archéologie dans l'Empire ottoman autour de 1900 : entre politique, économie et science, Bruxelles, 2004. 
Ce sont ces différents aspects que mettent en lumière plusieurs opérations documentaires, menées depuis plus de trois ans en collaboration par l'École pratique des hautes études (EPHE) et l'Institut national d'histoire de l'art (INHA), à l'initiative et sous la co-direction de M. François Queyrel, directeur d'études à l'EPHE, spécialiste de sculpture grecque. Elles offrent notamment aujourd'hui au public une base de données appelée "Nouveau Mendel », une exposition intitulée "Éclats d'antiques : sculpture et photographie, Mendel à Constantinople il y a un siècle " (Paris, INHA, 24 avril-20 juillet 2013), ainsi qu'un livre portant le même titre ${ }^{2}$, qui éclaire la genèse de l'œuvre.

\section{Une œuvre, un musée et des hommes}

Avec plus de 1800 pages et 1413 notices, le catalogue témoigne d'une véritable ambition scientifique. Salomon Reinach, d'autant plus à même de juger de la valeur de l'opus qu'il avait lui-même publié un guide du musée en $1882^{3}$, le considérait comme "le plus complet - avec celui du Vatican par M. Amelung - qui ait encore été publié d'une collection de marbres antiques [...] ; un ouvrage destiné à être consulté par des générations d'archéologues, qui met au point d'une façon définitive le travail de la génération précédente [...]. On reste confondu de la somme de travail que l'auteur a fournie et aussi de sa perspicacité, car ces monuments étaient loin d'avoir tous été publiés et il lui a fallu, pour nombre d'entre eux, faire œuvre personnelle d'exégète. L'ensemble forme [...] un répertoire archéologique de premier ordre, fait d'ailleurs pour être consulté dans le cabinet plutôt que sur place ; mais il sera facile d'en extraire, à coups de ciseaux, la matière d'un catalogue sommaire pour les visiteurs ${ }^{4}$ ".

La comparaison de Reinach souligne l'exemplarité de la publication, digne d'égaler la science allemande, ainsi que la valeur du musée de Constantinople. Le développement rapide de ce dernier reflète en effet l'évolution de la capitale de l'Empire ottoman, de plus en plus ouverte, au tournant du $\mathrm{Xx}^{\mathrm{e}}$ siècle, au mode de vie, aux sciences et aux techniques occidentales. À partir de l'implantation des collections d'antiques à Çinili Köş en 1875-1876, le développement rapide du musée est lié à la personnalité d'Osman Hamdi Bey (1842-1910) qui le dirigea de 1881 à sa mort. Agrandi entre 1891 et 1907 par de nouveaux bâtiments néo-classiques et sans cesse enrichi de découvertes archéologiques, grâce à la réglementation sur les antiquités trouvées en territoire national mise en place par Hamdi Bey dès 1884, le musée stambouliote devient une

2 M. Poulain - F. QueYrel - G. Paquot (dir.), Éclats d'antiques. Sculpture et photographie : Gustave Mendel à Constantinople, Paris, 2013, 307 p. Référence désormais abrégée en Éclats d'antiques; les mentions " (cat.) " renvoient aux notices de ce catalogue de 2013.

3 S. ReInach, Catalogue du Musée impérial d'antiquités, Constantinople, 1882.

4 Id., Revue archéologique, 1914, 2, p. 172-173. 
institution de premier plan ${ }^{5}$. Dans le même temps, tout au long du dernier tiers du $\mathrm{XIX}^{\mathrm{e}}$ siècle et de la première décennie $\mathrm{du} \mathrm{XX}^{\mathrm{e}}$, les puissances occidentales s'efforcent de multiplier les fouilles en Méditerranée orientale, poussant leur champ d'action jusqu'en Mésopotamie. Face à la puissance de l'archéologie allemande, la France parvient, tant bien que mal, à se faire une place, en grande partie grâce à Hamdi Bey et à son frère Halil Edhem (1861-1938), qui lui succède à la tête des musées jusqu'en $1931^{6}$. Ottoman francophile - il a fait des études supérieures en France où il a délaissé le droit pour les beaux-arts -, peintre orientaliste, archéologue de terrain (fouilles de Sidon en 1887, publiées avec Théodore Reinach, puis du sanctuaire d'Hécate à Lagina avec Joseph Chamonard, en 1892-1893), Hamdi Bey fait volontiers appel, pour réorganiser et publier les collections d'antiques, à des membres de l'École française d'Athènes : Salomon Reinach, André Joubin (dans les années 1890) ${ }^{7}$ puis Gustave Mendel, officiellement à partir de 1903.

Ce dernier poursuit sa mission à Constantinople pendant plus de dix ans, jusqu'à la Grande Guerre, malgré les difficultés de sa position et les problèmes financiers récurrents. Mendel doit en effet attendre 1910 pour se voir octroyer le titre de conservateur des Musées impériaux, et peine à percevoir son salaire, en principe payé conjointement par l'État français et le gouvernement ottoman ${ }^{8}$. C'est grâce à la ténacité et au soutien indéfectible des deux frères turcs que Mendel continue tant bien que mal à travailler sur les collections archéologiques. Ses qualités personnelles expliquent aussi qu'il ait fait l'unanimité, tant du côté français que du côté stambouliote. Théodore Homolle, directeur de l'École française d'Athènes dans les années où Mendel y séjourne en tant que membre, écrit à son propos dès 1902 que, outre son expérience scientifique, "il travaille avec une ardeur et une conscience parfaites, il est intelligent, fin, sait voyager, connaît les monuments, les inscriptions et a une grande culture ; je me ferais scrupule de ne pas ajouter que c'est un caractère d'une rectitude, d'une sûreté, d'une délicatesse rares. [...] Il est l'homme de cette place par son tact et son dévouement aux intérêts français ${ }^{9} "$. Douze ans plus tard, son sentiment n'a pas varié, tel qu'il l'exprime à l'Aca-

5 Pour plus de détails, voir Z. Kiziltan, «Dünden Bugüne, Istanbul Arkeoloji Müzeleri / Les musées archéologiques d'Istanbul, d'hier à aujourd'hui ", in Éclats d'antiques, p. $22-41$.

6 L.A. MeYER, "In memoriam : Halil Edhem Eldem (1861-1938)", Ars islamica 6 (1939), p. 198-201. Il était entré au service du musée dès 1889 .

7 Avant Mendel, et après le guide de S. Reinach, A. Joubin a ainsi publié quatre petits catalogues du Musée impérial ottoman, dont un Catalogue des sculptures grecques, romaines, byzantines et franques (Constantinople, 1893, 82-viii p.).

8 À partir d'archives ottomanes inédites, E. Eldem, professeur à l'université de Bogaziçi à Istanbul, complète, dans un article intitulé "Gustave chez les Turcs " (in Éclats d'antiques, p. 111-129) ce que l'on pouvait connaître de la mission de Mendel à partir des archives administratives françaises.

9 Archives nationales, F17/13598, lettre fragmentaire de T. Homolle, s. d. [= rapport 1902]. 
démie des Inscriptions et Belles-Lettres : Mendel est selon lui « l'un des promoteurs et le meilleur ouvrier de l'entente archéologique qui a rouvert à nos savants l'Asie Mineure et la Syrie, dont ils s'étaient depuis trop longtemps détournés ${ }^{10}$ ». Une demande de Légion d'honneur émanant du ministère de l'Instruction publique souligne encore les vertus du conservateur : "Les Catalogues qu'il a heureusement entrepris de plusieurs séries (bronzes, sculptures, etc.) des musées impériaux, où il a été attaché dès 1903 à la demande de Hamdi-bey, sont une œuvre de haute valeur scientifique ; elle fait le plus grand honneur à l'archéologie française et ne peut que contribuer à lui faire reconquérir la place qui lui est due et qu'elle occupait, il y a une vingtaine d'années dans les pays ottomans ${ }^{11}$. " Conformément à la pratique de l'archéologie sous la III ${ }^{\mathrm{e}}$ République, le rôle de Mendel ne se limite pas à la rédaction de catalogues : le missionnaire sert de correspondant et de trait d'union entre sa nation d'origine et l'Empire ottoman. Des archives et lettres conservées, il ressort que celui-ci fournit aux savants et aux ministères parisiens des nouvelles et des rapports archéologiques, ainsi que des propositions d'achat pour le Louvre (cat. 49 : Bibl. Institut de France, Ms 5773, fo 315-316) ; il se montre "fort utile aux savants français envoyés en Orient ${ }^{12}$ ", encourage l'octroi de fouilles à son pays natal et participe lui-même à des chantiers, notamment à Aphrodisias ${ }^{13}$. Quant à sa production scientifique, avant les volumes de sculpture, Mendel est l'auteur de deux autres catalogues - ne portant aucun nom d'auteur ni sur la page de titre ni sur la couverture - : Musées impériaux ottomans. Catalogue des figurines grecques, de terre cuite (Constantinople, $1908^{14}$ ) et Musées impériaux ottomans. Catalogue des sculptures grecques, romaines et byzantines du musée de Brousse (Athènes, 1908) ${ }^{15}$. Par ailleurs, il

10 T. HomOlLe, CRAI 1914, p. 522-524.

11 Archives nationales, F/17/17243 : Asie Mineure, dossier « Gustave Mendel 1903-1920 », lettre du ministère du 6 décembre 1912.

12 Archives MAE, Turquie 396 "Fouilles archéologiques V. juillet 1912-septembre 1915 ", note de Bayet, directeur de l'Enseignement supérieur au MIP, s.d. [circa novembre 1912].

13 G. Mendel, "Seconde note sur les fouilles exécutées à Aphrodisias par M. Paul Gaudin. Campagne de 1905 ", CRAI 1906, p. 158-184. La Bibliothèque de l'Institut conserve les originaux des deux plans publiés dans cet article (Ms 1901) : 2 documents datés de 1905 (cat. 61-62). Une annotation au crayon (apparemment postérieure ; de la main du donateur ou d'un bibliothécaire ?) les attribue à " Replat et Mendel » : d'après l'ordre des noms (non alphabétique) et à lire l'article dans lequel ils ont été publiés, il semble plutôt que c'est l'architecte Replat qui est l'auteur principal de ces dessins - suivant une traditionnelle répartition des tâches sur les chantiers archéologiques.

14 L'ouvrage est illustré de photographies signées " H. Demoulin, sc. ", reproduites sur des planches en fin de volume. Sur ce catalogue, voir I. Hasselin Rous, in Éclats d'antiques, p. $95-110$.

15 Les remerciements qui ouvrent cet ouvrage donnent quelques indications sur les outils bibliographiques dont Mendel a pu disposer à Constantinople, par ses contacts avec des Allemands, des Russes et des Pères Augustins de l'Assomption. Sur ce catalogue, voir également E. ELDEM, art. cit., p. 117-118. 
avait déjà contribué à la publication, en " collaboration discrète ", des fouilles de Tralles menées par le propre fils de Hamdi Bey, Edhem Bey, en $1902^{16}$.

\section{Photographies et dessins}

Dans les deux catalogues cités précédemment, figuraient des photographies d'objets, en nombre limité. Pour les trois volumes dédiés aux sculptures du musée de Constantinople, le parti est pris de reproduire de très nombreux dessins, insérés dans le texte. Salomon Reinach pour sa part estime que ces "images, dessinées à la plume ${ }^{17}$ d'après des photographies, sont très satisfaisantes et les critiques que j'ai entendu énoncer à leur sujet ne sont nullement fondées. Il est évident qu'elles ne donnent pas autant de détails que des photographies, mais elles sont autrement claires et aident beaucoup mieux à suivre les descriptions ${ }^{18}$ ". Une brève mention, dans une lettre de Mendel à Léon Heuzey du 15 juillet 1911 (cat. 51 ; Bibl. de l'Institut, Ms 5773, fo 320-321), évoquant l'accord " au prix très réduit convenu » avec le dessinateur explicite ce choix. En effet, en ce début du xx siècle, la technique de la zincographie s'avère beaucoup plus économique que la reproduction de photographies en héliogravure, qui ne peut se faire qu'en planches distinctes ${ }^{19}$. Les prises de vue ne sont cependant pas écartées de la publication, comme l'explique Homolle qui semble bien informé de la manière dont s'est déroulé le travail : "Les reproductions sont simples, mais consciencieusement exécutées par M. Payraud, surveillées de si près par M. Mendel, qu'elles rendent avec une louable fidélité non seulement l'aspect, mais aussi le style des modèles. On remarquera d'ailleurs que, pour donner toute garantie aux études archéologiques, l'administration des Musées [de Constantinople] met à la disposition du public des photographies, dont le numéro est indiqué à la suite de chaque article et dont on peut se procurer des épreuves ${ }^{20}$. " Le dessinateur Georges Payraud, bien que peu connu, est coutumier des dessins de sculptures antiques et médiévales : il semble en effet avoir collaboré avec Salomon Reinach pour son Répertoire de reliefs grecs et romains ${ }^{21}$,

16 Fouilles publiées et communiquées au monde savant par l'intermédiaire de Salomon Reinach : EDHEM-Bey, "Fouilles et découvertes à Tralles ", Revue archéologique 1904, 2, p. 348-362.

17 Cf. Mendel, Catalogue, I, p. xxi.

18 S. REINACH, Revue archéologique, 1914, p. 172.

19 J. Delatour, "La fabrique des images ", in Éclats d'antiques, p. 141-149.

20 T. HomOlLe, CRAI 1914, p. 523.

21 S. ReInACH, Répertoire de reliefs grecs et romains, Tome 1er, Les ensembles, Paris, 1909, p. ii, 360 et 363 : dessins sur la colonne Trajane. 
et a illustré l'ouvrage - moins scientifique - du Dr Gustave-Joseph Witkowski, L'art profane à l'église 22 .

Ce n'est que tout récemment que l'on a pu opérer le rapprochement entre les dessins des catalogues et les photos des antiques du musée, réalisées à cette époque par le célèbre studio stambouliote Sébah \& Joaillier. Des ensembles de tirages figurent ainsi dans les collections de l'inHA et de l'epHe. La Bibliothèque de l'inHA a pour origine la Bibliothèque d'art et d'archéologie constituée par le couturier et mécène Jacques Doucet (1853-1929) ; c'est à ce fonds original qu'appartient un exemplaire du Catalogue de Mendel, offert par son auteur (cat. 47), ainsi qu'un ensemble de 1300 photographies Sébah \& Joaillier sur la Turquie - dont on ignore en revanche la voie exacte d'entrée dans les collections -, incluant des vues des sculptures cataloguées par Mendel. Plus tard, André Joubin (1866-1944) ${ }^{23}$ - qui, comme on l'a vu, travailla également auprès de Hamdi Bey à Constantinople -, devenu premier bibliothécaire de la bibliothèque Doucet après le legs de celle-ci à l'université de Paris, y déposa ses papiers qui comprenaient quelques photographies supplémentaires de la même série du musée d'Istanbul. L'autre ensemble provient quant à lui de Gabriel Millet (1867-1953), directeur d'études à l'EPHE, qui parcourut durant quarante années les Balkans et le Proche-Orient à la recherche de monuments, manuscrits et miniatures d'époque byzantine. Il a réuni une importante documentation iconographique, aujourd'hui conservée au sein de la même institution sous la dénomination de "Photothèque Millet ». On y trouve deux beaux albums consacrés aux sculptures du musée archéologique d'Istanbul, réunissant des photos Sébah \& Joaillier ${ }^{24}$.

Ce studio réputé de Péra, le quartier européen d'Istanbul, résulte de l'association, vers 1890, de Jean Sébah (1872-1947), fils du photographe Pascal Sébah (1823-1886, installé dès 1857), et de Polycarpe Joaillier. L'atelier du père et de ses successeurs, apprécié de Hamdi Bey, diffusa de nombreux albums sur les monuments et la vie quotidienne ottomane, et contribua aux travaux archéologiques de l'orientaliste. À l'inverse des photographies touristiques, les vues du musée ne semblent pas avoir été largement diffusées, mais plutôt réservées à un cercle scientifique. On en trouve ainsi d'autres tirages dans les fonds de l'École française d'Athènes ou ceux de la Bodleian Library à Oxford $^{25}$. Les clichés originaux ont disparu, et à ce jour il n'existe pas d'archives Sébah $\&$ Joaillier. Les circonstances de la commande relative aux sculptures et sa réalisation

22 L'art profane à l'église : ses licences symboliques, satiriques et fantaisistes. Contribution à l'étude archéologique et artistique des édifices religieux, ouvrage illustré... avec le concours de G. A. Payraud, Paris, 1908, 3 vol. ; compte rendu critique par C. EnLART in Bibliothèque de l'École des chartes 70, 1909, p. 579-581.

23 Voir sa notice biographique dans È. Gran-Aymerich, Dictionnaire biographique d'archéologie 1798-1945, Paris, 2001, p. 367-368.

24 C. Jolivet-LÉVY - I. LAGOU, " Gabriel Millet et ses albums de photographies des sculptures du musée archéologique d'Istanbul », in Éclats d'antiques, p. 173-185.

25 Archives de l'EFA : fonds Ch. Picard 21, 2-6 ; Bod. Libr. : cote 014875678. 
sont donc mal connues. L'on peut seulement reconstituer que, comme il l'indique dans une lettre à Heuzey datée 13 août 1908 (cat. 50 ; Bibl. Institut de France, Ms 5773, fo 317-319), Mendel s'attache à ce que les objets soient pris de "face, profil, et revers »ce qui ne transparaît pas dans les dessins de l'ouvrage, ni dans les photos conservées qui montrent le plus souvent des vues de face et de profil $^{26}$. Le rapprochement des photos originales et des dessins a permis de vérifier le procédé décrit par Reinach : Peyraud a le plus souvent opéré un décalque de la photo correspondante (voir essai effectué fig. 1), et il suffisait ensuite aux éditeurs de réduire le décalque pour l'impression ${ }^{27}$. Ce souci de systématisation des prises montre la démarche scientifique du savant désireux d'appréhender tout le matériel sous les mêmes angles et d'en donner une vision systématique et sérielle; parallèlement, l'usage qu'il fait de la photographie indique qu'il la considère comme un moyen utile pour appréhender le matériel archéologique, même si elle n'est pas reproduite en tant que telle dans la publication finale.

\section{Le projet documentaire et pédagogique du centenaire}

À l'occasion du centenaire du Catalogue, tout ce patrimoine est l'objet d'une revalorisation, à la fois par des procédés traditionnels (exposition, publication) et par le recours à de nouvelles technologies numériques.

En premier lieu, le Nouveau Mendel permet, par le biais d'Internet, l'accès à une œuvre parfois difficile à consulter en bibliothèque aux lecteurs spécialistes de l'Antiquité, aux amateurs des beaux-arts ou aux visiteurs du musée archéologique d'Istanbul. Outre la numérisation de l'ouvrage original, il a été opéré une illustration photographique inédite des sculptures, au moyen des prises de vue réalisées par le studio Sébah \& Joaillier et conservées par l'INHA et l'EPHE. La réunion de ces données en une base informatique se présente à l'utilisateur sous forme de notices, interrogeables par un moteur de recherche ${ }^{28}$. Cet outil est désormais consultable en ligne via le serveur Adonis, à l'adresse suivante : http://alphaweb.tge-adonis.fr/mvcMendel/.

Le projet initial envisageait une confrontation de ces images anciennes avec des clichés actuels des mêmes objets, de manière à voir l'évolution de ces derniers en un

26 Exemple : la caryatide de Tralles (Mendel, Catalogue II, p. 257, notice 541) pour laquelle Mendel signale 5 photos (photographies $n^{\text {os }} 361$ : vue d'ensemble; 363 : tête de face, 624 : tête profil à droite; 362 : tête profil à gauche; 364 : revers de la tête), et dont on conserve la vue d'ensemble et le profil droit (voir la base de données Nouveau Mendel).

27 Cette éclairante expérience a été réalisée par J. Delatour (résumé dans « La fabrique des images ", p. 142). Sur les questions photographiques, voir également H. BOCARD, « De la fouille au musée : l'objet archéologique mis en scène ", in Éclats d'antiques, p. 151-161; E. ÖZENDES, From Sébah \& Joaillier to Foto Sabah. Orientalism in photography, Istanbul, 1999. Remerciements de Mendel à ses collaborateurs in Catalogue III, p. XI-XII.

28 Pour en savoir plus sur cette base de données : M. Bui, G. Paquot, A.-L. Pierre, F. QueYrel, "Le Nouveau Mendel », in Éclats d'antiques, p. 193-203. 
siècle d'intervalle (par exemple, certaines sculptures portaient des traces de polychromie, aujourd'hui disparues) et de bénéficier de commentaires actualisés (bibliographie récente, identification nouvelle, conservation...). Il faut espérer que cette base de données française sera dans l'avenir enrichie dans ce sens, grâce à des collaborations diverses - notamment avec les collègues turcs -, de manière à offrir un outil de recherches essentiel pour l'étude de ces collections.

De manière plus limitée, la genèse du Catalogue de Mendel est présentée au public sous la forme d'une petite exposition temporaire qui se tient à Paris, dans le bel environnement de la galerie Colbert de l'inHA. Le visiteur y est accueilli par la carte de l'Empire ottoman due à Henri Kiepert (Berlin, 1867 : hors cat.), qui est reproduite sur le sol. Cette carte permet de situer la provenance des antiquités dont il est question ici, et illumine par ses couleurs les murs couverts d'un choix de photographies anciennes sépia. Le paysage stambouliote est pour sa part représenté par un tableau de Félix Ziem - peintre voyageur (1821-1911) qui se trouve actuellement sous les feux des projecteurs par une rétrospective au Petit-Palais - montrant un Pin parasol à l'entrée du Bosphore au couchant (cat. 17 : Petit-Palais inv. PPP00242, vers 1857-1900), tandis que la ville elle-même et son urbanisme sont illustrés par un grand Panorama de Constantinople, pris depuis la tour de Galata entre 1872 et 1875 et constitué de dix épreuves photographiques assemblées formant une bande de plus de trois mètres de long (cat. 1 : Bibl. de l'inHA, coll. Jacques Doucet, 4 phot 038), ainsi que de vues du musée impérial redevables à Sébah \& Joaillier ou à d'autres éditeurs (cat. 6-7, 14, 81 : photos et cartes postales couleur). Au total ce sont environ cinquante photographies anciennes d'antiques qui sont exposées, appartenant aux collections Doucet de la Bibliothèque de l'inHA ou à la collection Millet de l'EPHE : certaines révèlent l'environnement des sculptures (salles ou jardin du musée : par ex. cat. 4 et 10) et les pratiques de prises de vue (tissus sombres tendus par un homme, Oriental ou Occidental : par ex. 15 et 43-44, voir fig. 2). Outre quelques imprimés (notamment les catalogues précédents de Reinach et de Joubin : cat. 45-46) et les albums Millet (sans $\mathrm{n}^{\circ}$ cat.), l'exposition montre quelques documents manuscrits : trois lettres de Mendel à Heuzey (cat. 49-51 : Bibliothèque de l'Institut) et des archives provenant de la famille d'Halil Edhem relatives à l'impression du troisième volume du Catalogue (cat. 52-54 : coll. Edhem Eldem). Quelques timbres et pièces commémoratives illustrent le prestige dont jouit, encore aujourd'hui, la figure d'Hamdi Bey (cat. 29-30).

L'attention du visiteur sera sans doute attirée par une pièce étonnante - placée au centre de l'exposition - qui a été remarquée par les premiers voyageurs français dès le milieu du XIX ${ }^{e}$ siècle. Gustave Flaubert et Maxime du Camp visitent en effet "le musée d'antiques " - alors une simple accumulation d'armes et d'objets divers à $S^{\text {te }}$-Irène - le lundi 18 novembre 1850 et notent la présence d'une «statuette de comédien ${ }^{29}$ ». Du

29 Voir les pages stambouliotes du Carnet de voyage de Flaubert, édité par P.-M. DE BiASI in Éclats d'antiques, p. 213-227. 
Camp aurait tenté, en vain, d'acquérir cette " chose curieuse » lors de sa mise au jour en octobre à Güzel-Hissar ; deux mois plus tard, à Constantinople, il fait réaliser en plâtre un moulage de cette statue-colonne romaine en marbre, représentant un "leno " qui fit l'objet d'un article de la part de Salomon Reinach en 1883 (cf. cat. 18-20, avec références et citations de Maxime du Camp). L'objet original, appartenant au musée ottoman, figure dans le catalogue de Mendel ; l'exposition montre, outre sa reproduction photographique par Sébah \& Joaillier, une copie en bronze, réalisée à la cire perdue par Eugène Gonon en 1881 pour Maxime du Camp à partir de son moulage et aujourd'hui conservée au Cabinet des Médailles (cat. 19 : BNF / Dép ${ }^{t}$ des monnaies, médailles et antiques, reg. F.4401).

À la manière d'un triptyque, la base de données, l'exposition de l'inHA et la publication qui l'accompagne, réunissant quant à elle, sous la forme d'un livre de la collection "Recherches " d'Armand Colin, seize contributions et le récapitulatif des objets montrés, permettent ainsi de reconstituer - à l'exception de quelques " éclats » d'histoire encore manquants - le puzzle d'une œuvre pérenne, née à la croisée de l'Occident et de l'Orient et dans le foisonnement scientifique, culturel et relationnel de l'archéologie de l'aube du $\mathrm{xx}^{\mathrm{e}}$ siècle.

\section{Informations pratiques / Récapitulatif}

- EXPOSITION Éclats d'antiques. Sculpture et photographie : Gustave Mendel à Constantinople. Organisée par l'EPHE et l'INHA, avec le soutien notamment du labex TransferS ; commissaires : Martine Poulain (directrice de la Bibliothèque de l'INHA) ${ }^{30}$ et François Queyrel (directeur d'études à l'EPHE), assistés de Gérard Paquot (chercheur associé).

24 avril 2013 - 20 juillet 2013 INHA, Rotonde de la galerie Colbert - Salle Longhi 2, rue Vivienne, 75002 Paris ( $\mathrm{M}^{\circ}$ Bourse ou Palais Royal). Du mardi au samedi $13 \mathrm{~h} 30$ - 18h30. Entrée libre.

- Site Internet de l'exposition, réalisé par Pierre-Olivier Védrine (EPHE)

http://www.expo-eclats.com/

- Page facebook de l'exposition

http://www.facebook.com/pages/Eclats-dantiques/356257824480692

- CONFÉRENCES accompagnant l'exposition (lieu : INHA - 2, rue Vivienne, 75002 Paris) :

- le 23 avril à $17 \mathrm{~h}$ : présentation de l'exposition par les commissaires de l'exposition, des auteurs du catalogue et des étudiants de l'EPHE;

30 Auteur du remarquable ouvrage Livres pillés, lectures surveillées. Les bibliothèques françaises sous l'Occupation, Paris, 2008 (bientôt réédité en format poche). 
- le 28 mai à $17 \mathrm{~h}$ : « Gustave Mendel et Osman Hamdi Bey ", par Edhem Eldem (université du Bosphore) ;

- le 12 juin à $17 \mathrm{~h}:$ : Tombes royales - tombes dynastiques d'Asie Mineure, un état des dernières recherches ", par Olivier Henry (Institut français d'études anatoliennes).

- CATALOGUE de l'exposition :

M. Poulain, F. QueYrel et G. Paquot (dir.), Éclats d'antiques. Sculpture et photographie : Gustave Mendel à Constantinople, Armand Colin, 2013, 307 p.

- BASE de DONNÉES « Nouveau Mendel » http://alphaweb.tge-adonis.fr/mvcMendel/

\begin{tabular}{l} 
Annick FENET \\
\hline UMR 8546 AOROC \\
Membre associée de PLH-ERASME (EA 4601) \\
Collaboratrice du centenaire du Catalogue de Mendel \\
86, rue Bobillot \\
F-75013 Paris \\
annick.fenet@ens.fr
\end{tabular}
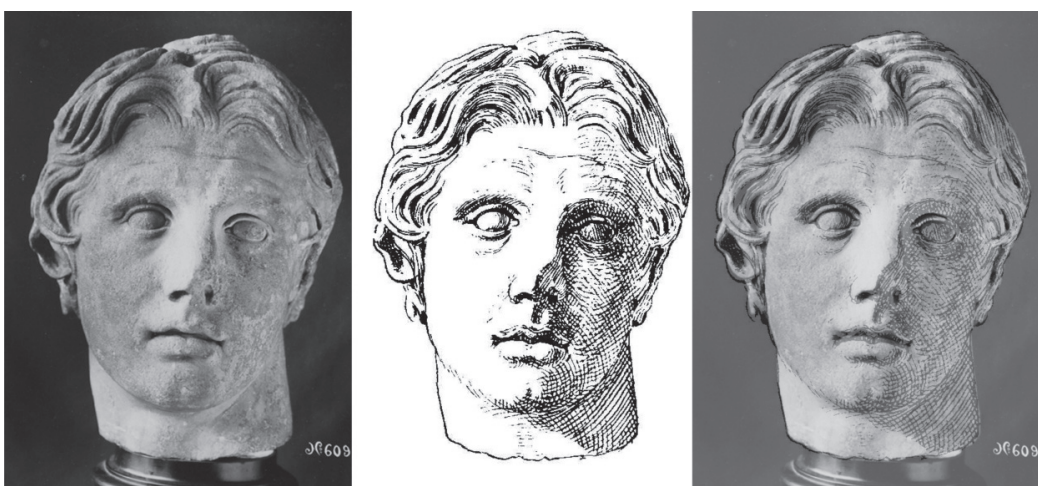

Fig. 1 : Tête d'Alexandre, de Pergame (notice Mendel 538). Résultat de la superposition (à dte) de la photo Sébah \& Joaillier (à g., (C) Bibl. de l'INHA, coll. J. Doucet, PAAGR II, 77, pl. 19) avec le dessin de Peyraud (au centre). 


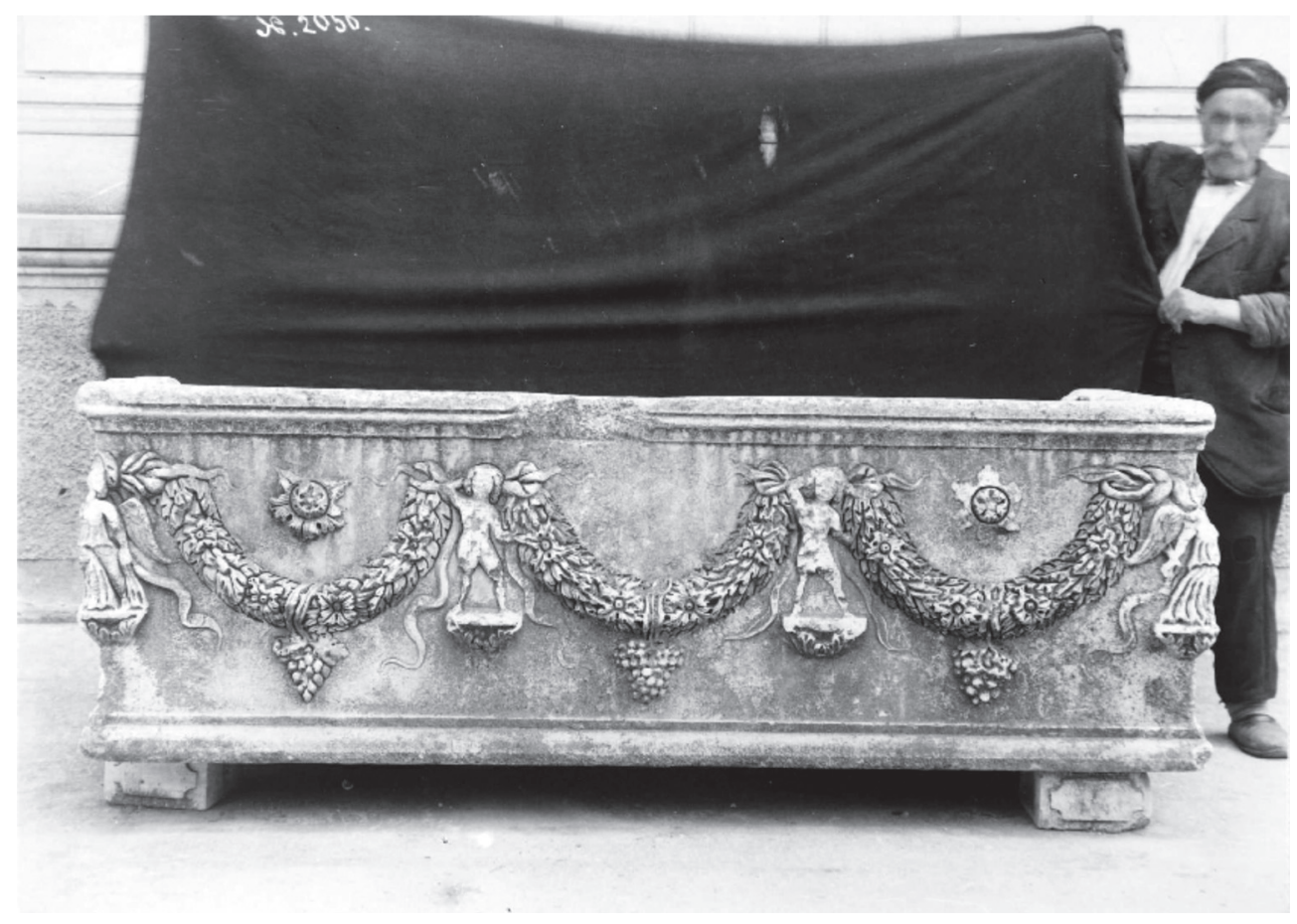

Fig. 2 : Sarcophage à guirlandes (notice Mendel 1160) : prise de vue avec un assistant tenant un drap sombre.

Photographie Sébah \& Joaillier (C) Bibl. de l'InHA, coll. J. Doucet, PAAGR II, 91, pl. 18. 